\title{
Atypical Coronary Artery Disease Symptom
}

National Cancer Institute

\section{Source}

National Cancer Institute. Atypical Coronary Artery Disease Symptom. NCI Thesaurus. Code C99917.

Sharp stabbing chest pain or reproduction of pain on palpation. Less common isolated presentations, primarily in older adults, include nausea and vomiting, diaphoresis, and unexplained fatigue. J Am Coll Cardiol, 2007; 50:1-157, doi:10.1016/j. jacc.2007.02.013 (Published online 6 August 2007). (ACC) 